\title{
Two-dimensional analysis of ice ridging in the Beaufort Sea using aerial photography
}

\author{
J. E. LEWIS, \\ Department of Geography, McGill University, Montreal, Quebec H3A 2K6, Canada \\ P. BudKeWITSCH, \\ Department of Earth and Planetary Sciences, McGill University, Montreal, Quebec H3A 2K6, Canada \\ G. Newton, \\ Department of Biology, University of Waterloo, Waterloo, Ontario, Canada \\ M. SAyed and R. M. W. Frederking \\ Division of Mechanical Engineering, National Research Council, Ottawa, Ontario, Canada
}

\begin{abstract}
Aerial photography was obtained for the Beaufort Sea north of Tuktoyaktuk. The flight path covered two distinct ice zones over a $15.5 \mathrm{~km}$ transect extending perpendicular to the coast, yielding fifty-nine photographs at a scale of $1: 2000$. The process of ridge extraction was automated using a series of computer algorithms for image filtering, edge detection and edge linking. Examples from two different sections along the transect are chosen for presentation: (a) a heavily ridged area, and (b) an area with one dominant linear ridge feature that separates ice cover of different age. Two parameters used in the automated process, a minimum edge gradient and minimum number of connected pixels said to form a continuous ridge segment, influence the number, length and spatial pattern of extracted ridges. Direct one-to-one correlations between manually interpreted ridges from photographs and the algorithm extracted ridges from digital data are not always possible. However, results indicate that the automated ridge extraction procedure reliably characterizes the overall direction and density of the ice ridges. The distribution of the ice-ridge directions is estimated from circular (angular) histograms constructed directly from the digital data. Analysis of the Beaufort Sea transect reveals that the ice ridging is strongly anisotropic, with a principal direction parallel to the local coastline.
\end{abstract}

\section{INTRODUGTION}

Ice-ridge investigations have been traditionally conducted on one-dimensional profiles or transects. While one-dimensional data supplies important information about the distribution statistics of ice ridges, it has not proved useful for explaining the two-dimensional characteristics of ice ridges or the dynamics of their formation. This paper reports on the effectiveness of an automated ridge identification procedure for extracting ice ridges from digitized aerial photographs, via a series of image processing algorithms. We compare these results with ridges identified manually by a photo interpreter.

The techniques developed by Newton and Kendrick (1990) for feature extraction in taxonomy, has been successfully applied to identifying geologic lineaments from digital satellite images (Newton and Budkewitsch, 1989). In this study, we have implemented these techniques and determined their optimal sequence and parameters for extracting the two dimensional form of ice ridges. The digital procedures presented are of a general nature and may be applied to Synthetic Aperture Radar (SAR) data or other types of digital images.

Other automated ridge and lead extraction techniques for sea-ice analysis have been reported in the literature. Samadani and Vesecky (1990) developed a maximum $a$ posteriori procedure with a multiplicative noise model for finding curvilinear features in speckled images. Vesecky and others (1990) used a threshold operation to identify bright regions on SAR images and then applied a linedetection operation using a local Kasvand operator (cf. Kasvand, 1974). Eppler and Farmer (1991) employed image-processing techniques to assess sea-ice types and lead features from SSM/I passive microwave data using a differential gradient operator in the row and column directions of the image. A plot of the circular variance was constructed to portray the geometric relationship between the row and column directions with its resultant magnitude and compass orientation. Similä and others (1992) approached the problem of ridge extraction for SAR images by a statistical analysis of the intensity histogram. From the SAR intensity data, they found that 


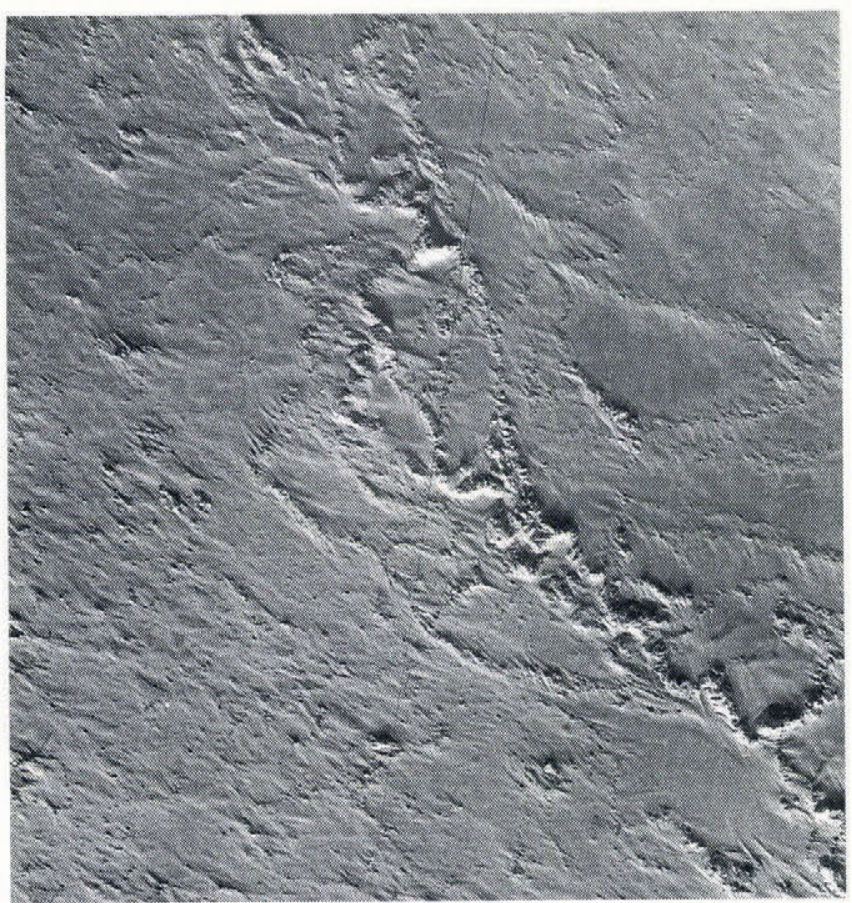

Fig. 1. Aerial photograph 1021 of the Beaufort Sea transect (March 1990). This region is characterized by a high density of prominent ridges in a northwest to southeast zone across the photograph.

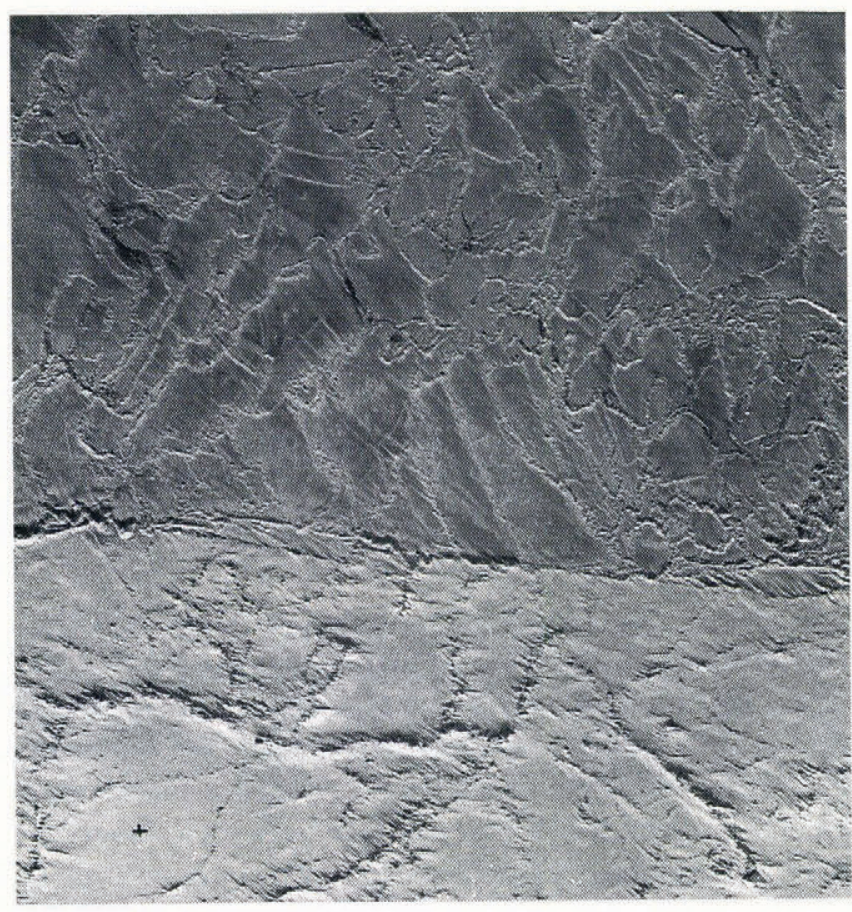

Fig. 2. Aerial photograph 1034 of the Beaufort Sea (March 1990). This region contains relatively thick cover ice in the south and thinner ice in the northern part. A sharp east-trending boundary marks the limit between the two. Ridging is present only in the southern part.

the tail-to-mean ratio with a threshold of $10 \%$ was a good predictor of ridge intensity.

Vertical aerial photography was obtained from the Beaufort Sea, north of Tuktoyaktuk, during March 1990. This photographic database offered an opportunity to develop procedures for recognition and extraction of sea ice ridges in their two-dimensional form. The photographic transect extended due north, along a line (starting at $133^{\circ} 01^{\prime} \mathrm{W}$ and $69^{\circ} 38.7^{\prime} \mathrm{N}$ ) flown perpendicular to the coast for a total continuous distance of $15.5 \mathrm{~km}$, of which $9.9 \mathrm{~km}$ was ice cover. Fifty-nine photographs provided the analogue data for our study at a scale of $1: 2000$. Ice characteristics were examined from images covering two distinct ice regions along the March 1990 transect - landfast and shear-zone ice regions.

In this paper we confine our discussion to two examples along the flight transect that display representative ridging characteristics: photo 1021 and photo 1034 (Figs 1 and 2). The region represented by photo 1021 has a high density of ice ridges in the shear zone. Photo 1034 is located about $2.5 \mathrm{~km}$ farther north, has a lower iceridge density, and shows a contact zone between first year ice of different ages. Using these two examples, we describe the automated procedure and the criteria used for extracting the ridges in digital form from the rasterized photographs. A complete statistical analysis of the entire transect for both the one- and two-dimensional ridge patterns and a comparison between the photo interpreted and algorithm extracted ice-ridge data are described by Lewis and Budkewitsch (1993).

\section{MANUAL PROCESSING OF AERIAL PHOTOGRAPHS}

From the series of photographs, 27 were selected and processed to eliminate overlap. For the manual procedure, linear features (ridges) from each photo were selected by a trained interpreter and traced by hand. Relative ridge heights were calculated photogrammetrically using a mirror stereoscope. Figures $3 \mathrm{a}$ and $6 \mathrm{a}$ illustrate the results of the manual interpretation of ridge distribution for photo 1021 and 1034. Spot heights measured at spacings corresponding to 20 to $30 \mathrm{~m}$ along the selected ridges range from 0.37 to $4.36 \mathrm{~m}$ in height. Based on two height verifications on each individual photograph, the relative error in estimating height was $10 \mathrm{~cm}$ or less.

\section{DIGITIZATION OF AERIAL PHOTOGRAPHS}

The automated extraction of ridge features from the aerial photographs required first that each photograph be converted to digital form. This was accomplished through the use of an optical scanner, which is a charged coupled device that produces a raster based on intensity, according to a calibrated grey scale. A Microtek ${ }^{\mathrm{TM}}$ flatbed scanner that produces up to 300 pixels per inch (ppi) and 256 grey-scale resolution was used for this purpose. Following calibration and testing different scales of resolution, we found satisfactory digital reproduction of the photographs for image processing at a resolution of $50 \mathrm{ppi}$, where each pixel corresponds to a $1 \times 1 \mathrm{~m}$ area. The digital file for each photograph requires about 250 kilobytes of storage, consisting of 8-bit values (integers from 0 to 255) stored for each row and column position. Each scanned photograph consists of approx- 
imately 500 rows by 500 columns and stored as a Tagged Image File Format (TIFF) file, an industry standard for digital images.

The images were cropped from their original scanned dimensions to eliminate borders and overlap between photographs. Due to irregularities in the flight path, some non-parallelism between images occurred, but this was minor (average of $2^{\circ}$ rotation), and consequently, less than $0.5 \%$ of the total rasterized area was repeated between images. No ridge information was duplicated or lost in the mosaicking process since there was approximately $10 \%$ marginal overlap between the digitized photographs where the seams between images could be chosen.

\section{PROCEDURE FOR DIGITAL IMAGE ANALYSIS}

Image processing is implemented through mathematical operations on the quantized grey-level values assigned to each pixel $(x, y)$ location on the image. The identification of ice ridges from digital images is possible because at the ridge location, the grey-level values are significantly different from the surrounding, ridge-free ice field. This is due to the fact that ice ridges stand out in relief and produce shadows, thus creating strong illumination contrasts. The procedure for obtaining the ice-ridge information is described below.

Extracting ice ridges from the background is accomplished through image-processing techniques called edge detection (see Martelli, 1972; Davis, 1975; Ballard and Brown, 1982). Three characteristics of the extracted ridges (edges), were recorded: (1) coordinates of pixels that delineate the ridges, (2) the gradient of the edge at the pixel, quantified by a Sobel filter (Giardina and Dougherty, 1988), and (3) the azimuth direction of the ridge at each pixel. Three processing steps were necessary to obtain the ridge characteristics from the digital images: (1) initial filtering, (2) edge detection, and (3) edge linking.

In order to reduce image noise, high-frequency filtering was performed with an edge-preserving adaptive (EPA) filter, developed for this study. The EPA filter is a local operator that selectively block-averages the image with a $3 \times 3$ kernel while preserving the edge information. Each pixel is averaged with five neighbouring connected pixels in the eight possible configurations. The eight convolutions are calculated for the centre pixel and the value from the convolutions which is closest to the original pixel value is stored as the new pixel value in the filtered image. A reduction of image noise was observed with each pass of the filter. In this study, two passes of the EPA filter adequately reduced the number of ridge identification errors.

The next step in the computing sequence determines the direction and magnitude of the grey level gradient at each pixel. These calculations are performed on the filtered images with a $3 \times 3$ Sobel filter which evaluates the local grey-level gradient. At each pixel, the Sobel edge strengths (abbreviated hereafter as edge strength) are calculated for the eight cardinal directions and the highest return value is stored. The pixel direction is defined as the azimuth orthogonal to the maximum local grey-level gradient. The angular values were determined to the nearest degree using real number calculations (Newton and Kendrick, 1990). For each pixel site, the edge-strength value and its direction are recorded.

The third step attempts to reduce unwanted edges (identification errors), such as those caused by snow drifts on the ice surface, by selecting only the pixels or edges which are associated with an actual ridge crest. Following the recommendations of Newton and Kendrick (1990), the edge feature of interest (the ice ridge) should possess the following properties:

(1) as the ridge is approached in a direction normal to its length, the edge strength should assume the maximum value;

(2) the pixel's immediate orthogonal neighbours should have the same edge direction $\left( \pm 45^{\circ}\right)$ as the original pixel.

The general implementation of this method enables the mapping of the two-dimensional form of the ice-ridge fields. However, for the specific case, two criteria must be considered when selecting possible pixels as ridge candidates:

(1) a minimum Sobel cut-off value, $\mathrm{S}_{\mathrm{v}}$;

(2) a minimum number of pixels that are in contact along their sides or corners.

In this study, the minimum edge strength, $\mathrm{S}_{\mathrm{v}}$, was determined for each of the 27 images. A standard $S_{v}$ was not possible since illumination of the ridge-free ice field was variable during acquisition of the photography. Most of the area represented in each photograph is ridge-free ice and therefore the modal grey level is a good measure of the ice-field illumination intensity. The $\mathrm{S}_{\mathrm{v}}$ for each image was determined using the following empirical relationship:

$$
\mathrm{S}_{\mathrm{v}}=270-\text { mode }
$$

Pixels that meet the minimum cut-off (criterion 1) are stored in a list. This list of candidate ridge sites is sorted into strings of connected pixels whose high edge strengths and directions are conformable to a linear or curvilinear edge and thus have a high probability of being part of an actual ice ridge. Examination of these candidate pixels indicate that many linear or curved features consisting of less than 10 connected pixels were features such as snow drifts and were clearly not ridge structures. The minimum length of connected pixels (criterion 2) used to characterize potential ridge structures therefore, was 10 . This minimum length was kept constant for all the images of the transect, which corresponds to a ground distance of about 10 to $14 \mathrm{~m}$. The end product now is a segmented image, identifying the ridges as linear forms with quantifiable attributes in geographic space.

The directional character of the identified ridges, is displayed as a circular histogram (see Figs 5d and 6d, discussed below). The statistics accompanying the diagrams are based on the theory developed by Robin and Jowett (1986) applied to angular data. The direction of each ridge segment was estimated as the peak value of the contoured angular data. The directions of each constituent pixel are weighted by the mean edge 
strength, and contoured using a Gaussian function (cf. Robin and Jowett, 1986; Newton and Budketwitsch, 1989).

\section{RESULTS AND DISGUSSION}

The two example areas chosen from the 27 photographs along the transect depict contrasting forms of ice-ridge structures. Photograph 1021 (Fig. 1) displays a complex ridge structure with ice of the same age, whereas photograph 1034 (Fig. 2) shows two distinct ice fields of different thickness with a sharp ridge-like boundary along the ice-ice contact zone.

\section{Photograph 1021 - heavily ridged region}

The manual interpretation of the ridge structure for this photograph is shown as Figure 3a. In this region, ice ridging occurs along a complex curvilinear zone, extending from the southeast to the northwest. For this interpretation, no a priori minimum height was set, however the minimum recorded spot height is $0.37 \mathrm{~m}$. The locations and values of the spot heights shown in Figure 3a have an average height of $1.98 \pm 1.24 \mathrm{~m}(\mathrm{n}=$ 83). The greatest measured ridge height is $4.36 \mathrm{~m}$ and lies along the main axis of the ridge structure. A total of 23 ridges are interpreted, of which 16 have a length greater than $30 \mathrm{~m}$. The maximum ridge length is approximately $250 \mathrm{~m}$.

The computer-generated ridge forms are highlighted as white pixels and shown in Figure 3b, superimposed on the digitized grey-level image of the aerial photograph. Ridge forms in this image have values of $\mathrm{S}_{\mathrm{v}}$ exceeding 120 and a minimum ridge length of 10 pixels. The frequency distribution of the edge strengths (Fig. 4), decreases in a manner similar to a negative exponential distribution.

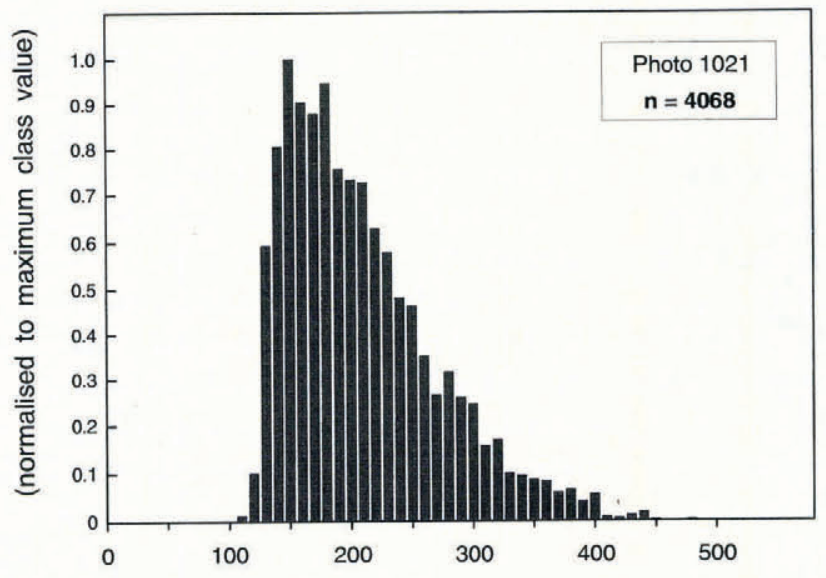

Fig. 4. Frequency histogram of Sobel values for all pixels selected as ridges, shown in Figure 3b. Maximum class contains 339 pixels.

The mean edge strength is $213 \pm 63$ ( $\mathrm{n}=4068)$ for the pixels that meet the criteria of the automated method. A total of 225 separate ridges or ridge segments are identified, with an estimated mean length of $23 \mathrm{~m}$ and a maximum length of approximately $80 \mathrm{~m}$.

Whereas subjective visual comparisons can be made between the algorithm-extracted ridges and those interpreted (Fig. 3a,b), obvious differences exist. The automated procedure extracts the fine scale structure in the ridge pattern rather than defining an integrated pattern as determined by the photo interpreter. In Figure $3 \mathrm{~b}$, ridges that have heights greater than $0.7 \mathrm{~m}$ above the ice surface are generally distinguished from the background. This is close to the subjective cut-off height used by many investigators characterizing ridge statistics in Arctic sea-ice studies. The exception to this effective cutoff height can be seen in the southwest quadrant of the

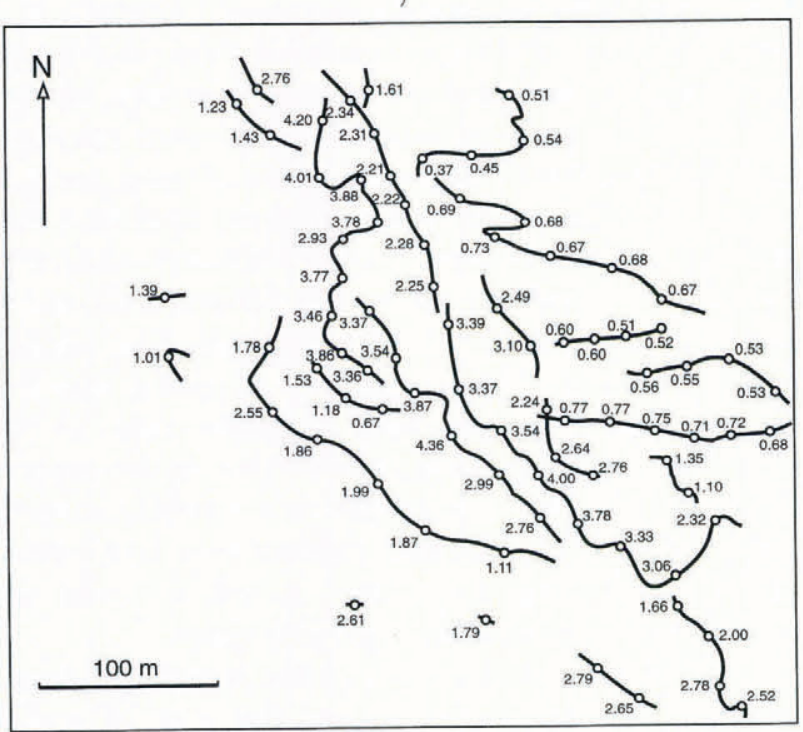

a

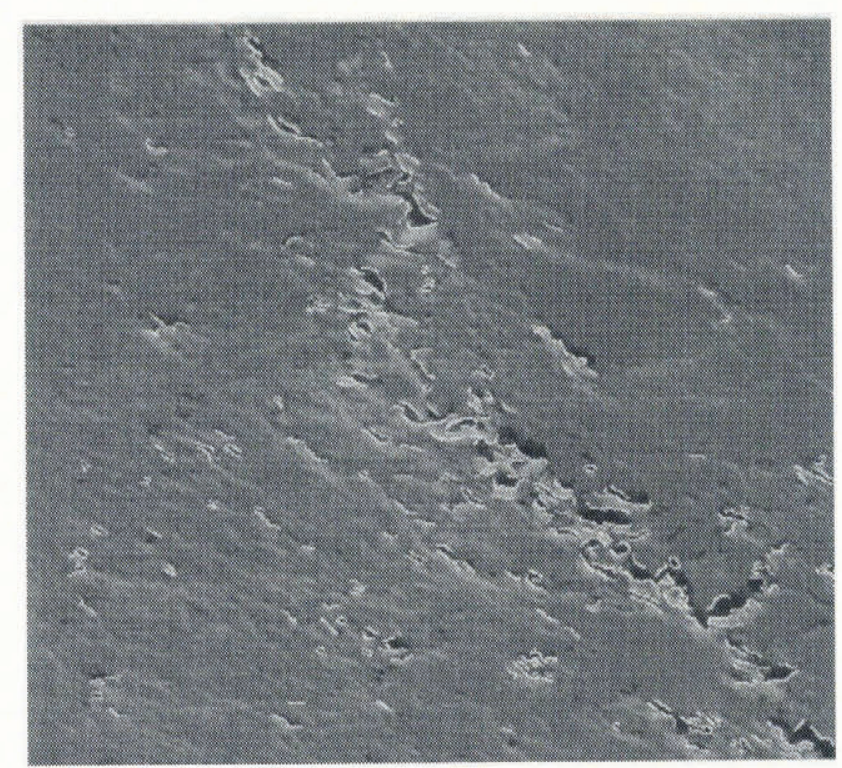

b

Fig. 3. Photo 1021. a, Interpretation of the ridge pattern for photo 1021 by photo interpreter. Measurements of ridge heights are given in metres. $b$, Automated ridge detection overlain (white) on the digital grey-scale image. The Sobel cut-off value is 120 and the minimum ridge-length criteria is 10 pixels $($ about $10 \mathrm{~m})$. Pixel resolution is $1 \times 1 \mathrm{~m}$. 

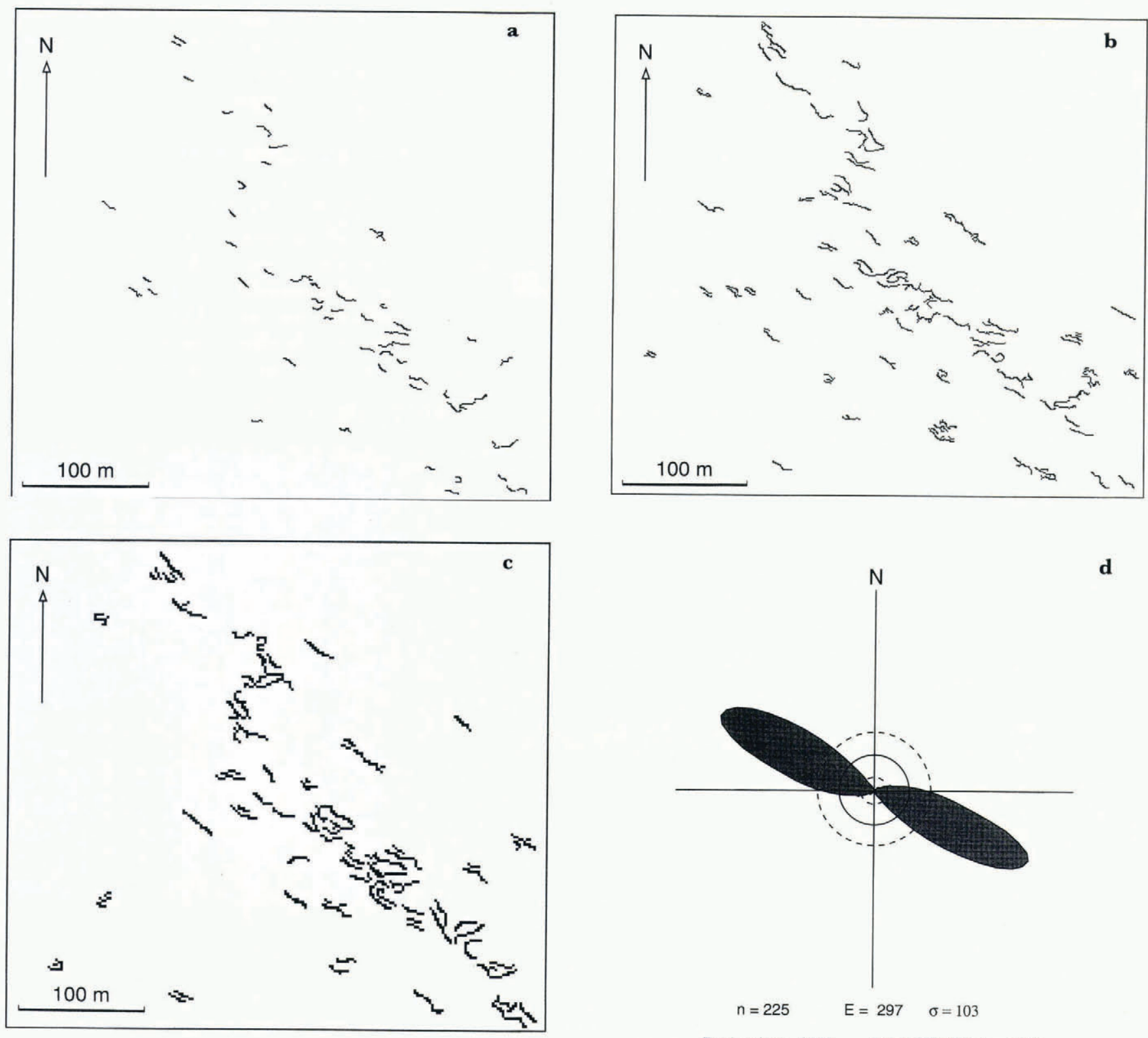

d

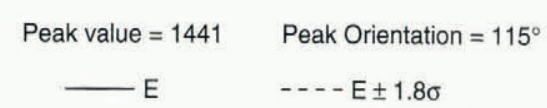

Fig. 5a-d. Automated ridge-detection of photo 1021. a, Using a higher Sobel cut-off value $\left(S_{v}=200\right)$ and maintaining the minimum ridge-length criteria of 10 pixels. This pattern is a subset of those ridges shown in Figure $3 b . b$, Maintaining the original Sobel cut-off value of 120 and raising the minimum ridge-length criteria to 20 pixels. This pattern represents a subset of the ridges found in Figure 3b. c, Using a lower resolution $(2 \mathrm{~m})$. The Sobel cut-off value is 120 and the minimum ridge length displayed is 10 pixels (about $20 \mathrm{~m}) . d$, Circular histogram of ridge directions. $E$ is the expected frequency of an isotropic distribution of ridges (solid circle). A significant anisotropy of the ridges in the $115^{\circ}$ direction is indicated by the peak value exceeding $E+1.8 \sigma$ (dashed circle).

photograph where a rough surface texture of the ice field exists. This is caused by elongated snow drifts, which produce prominent shadows resulting in false ridge identifications by the automated method. Such features are not classified as ice ridges by a knowledgeable photo interpreter.

A brief comparison can be made to illustrate the effect of variation in the two criteria used to quantify the ridge character. Figure 5 a represents the ridge forms extracted with a higher cut-off value $\left(S_{v}=200\right)$, but similar 10 pixel minimum length as used in Figure $3 \mathrm{~b}$. The higher minimum edge strength eliminates some of the misidenti- fied "ridges" in the southwest quadrant. On the other hand, it also reduces some ridge information in the main southeast to northwest ridge structure which runs across the image. In Figure $5 \mathrm{~b}, \mathrm{~S}_{\mathrm{v}}$ is the same as in Figure $3 \mathrm{~b}$, and the minimum ridge length is doubled to 20 pixels (about $20 \mathrm{~m}$ ). Fewer ridges are extracted by increasing the minimum length criteria, but numerous ridges are still identified along the main axis of the ridge structure.

If we examine the digitized aerial photograph at a lower resolution, some of the higher frequency edges are subdued. This is another parameter which improves the elimination of edges not significant to the study. Figure $5 \mathrm{c}$ 
is a resampled image of photo 1021 which has a new pixel resolution of $2 \times 2 \mathrm{~m}$. The same statistical distribution of grey levels allows, according to Equation (1), the same $\mathrm{S}_{\mathrm{v}}$ to be used as in Figure $3 \mathrm{~b}$. Selecting a minimum ridge length of 10 pixels is approximately equivalent $20 \mathrm{~m}$ and therefore the criteria used in Figure $5 \mathrm{c}$ are comparable to those that produced Figure 5b. By inspection, it can be seen that lowering the resolution prior to ridge extraction also eliminates most misclassified "ridges" found in the southwest quadrant.

Additional information about the overall ridge structure can be obtained from a plot of the direction and magnitude of the ridges in the form of a circular histogram (Fig. 5d). A strong anisotropic character of the ridging process in the $115^{\circ}$ direction is clearly indicated. The peak value in the principal direction substantially exceeds the level of significance $(\mathrm{E}+1.8 \sigma)$, indicated by the outer dashed circle on the diagram.

\section{Photograph 1034 - Ice contact zone}

The photograph (Fig. 2) of this region displays a sharp linear contact zone with ridging occurring between the thin ice that formed over an open lead to the north and the older ice cover on the south side. The ridge pattern interpreted manually is shown as Figure 6a. Fewer ridges compared to 1021 are identified by both the automated method and by photo interpretation. Seven ridges are

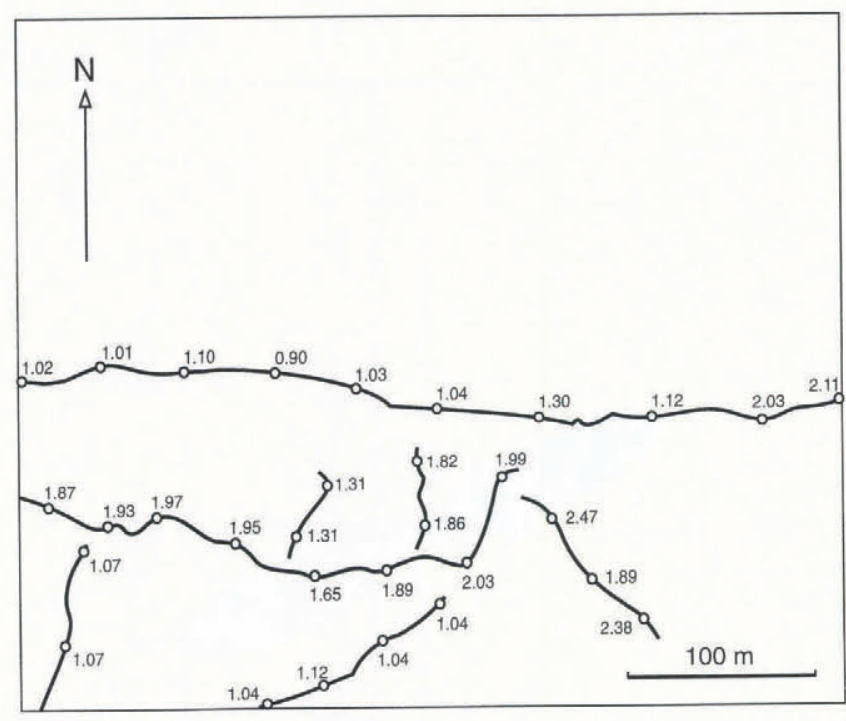

a

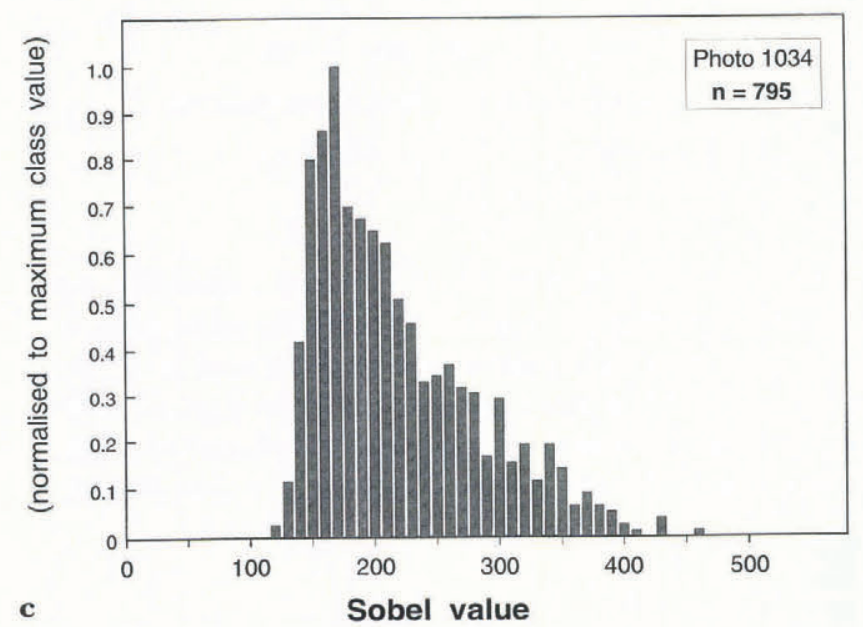

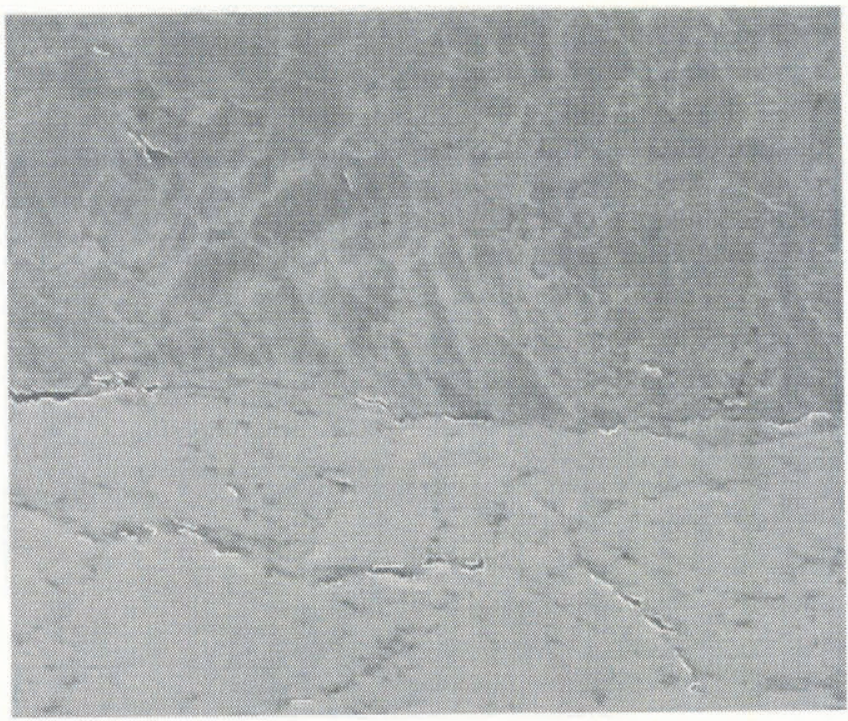

b

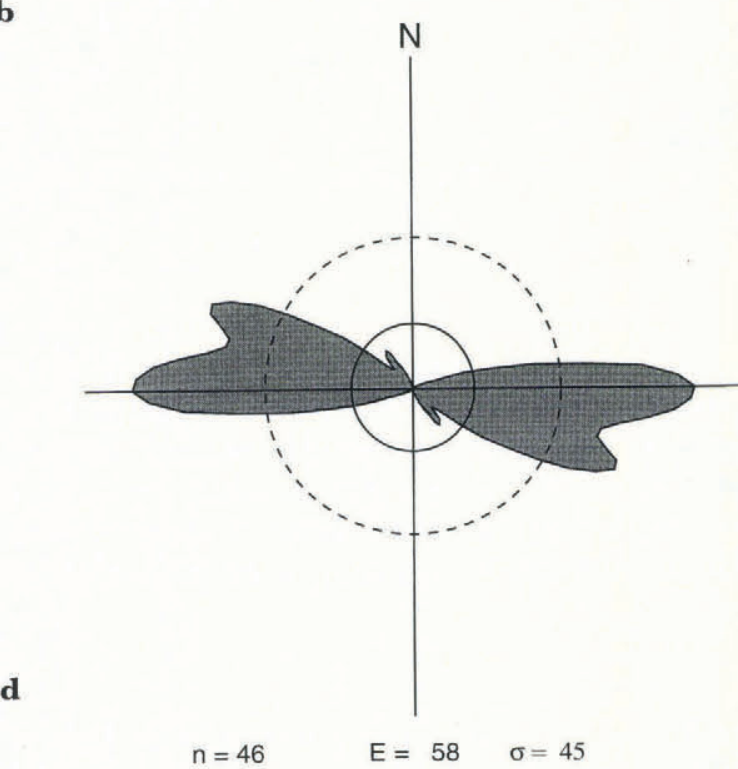

Peak value $=264 \quad$ Peak Orientation $=90^{\circ}$ 
designated with a mean spot height of $1.53 \pm 0.48 \mathrm{~m}(\mathrm{n}=$ 31 ) and the highest ridge measured is $2.38 \mathrm{~m}$.

For the automated analysis, $\mathrm{S}_{\mathrm{v}}$ was found to be 130 , according to Equation (1), and as before, we found that a minimum of 10 connected pixels best characterized the ice ridges. Figure $6 \mathrm{~b}$ illustrates the distribution of ridges extracted from this photo, which resulted in detecting 795 pixels in a total of 46 individual ridges or ridge segments. The frequency distribution of the edge strengths (Fig. 6c), exhibits similar statistics to the distribution in Figure 4. The mean edge strength of $203 \pm 61(n=795)$, mode and range of values recorded are somewhat lower, but not significantly different from 1021 or the other images examined along the transect.

The circular histogram for this photograph (Fig. 6d) shows a dominant east-west $\left(90^{\circ}\right)$ trend representing the well-defined ridge boundary developed across the ice field. A significant secondary lobe at $112^{\circ}$ can be accounted for by the few ridges present south of the boundary zone. It should be noted that this secondary direction is similar to the direction observed in photo 1021 which lies farther south.

\section{LIMITATIONS}

There are two analytical problems related to the digital procedure distinguishing ice ridges from the background image. One problem with aerial photography is that the natural light source of the Sun has an elevation and incidence angle which suppress the expression of linear features in positive relief that are parallel to the Sun azimuth. On the other hand, the visibility of similar structures orientated at high angles to the Sun azimuth are greatly enhanced (through shadow casting). This difference is further accentuated at low Sun elevations, a problem particular to the Arctic latitudes. The photographs show the Sun azimuth to be approximately due south, providing good contrasts for most ridge orientations, except in a north-south direction. Image processing and ridge extraction from the digital images relies largely on an illumination contrast. In photo 1034, for example, three relatively short north-south trending ridges identified by the interpreter (Fig. 6a) were omitted from the data set by the automated approach (Fig. 6b).

Another limitation is that a small number of non-ridge related features are being classified as ridges by our automated procedure, such as snow drifts in photo 1021 (Fig. 3b), and a few small leads in the younger, thinner ice, north of the boundary zone in photo 1034 (Fig. 6b). In regions of thin ice, strong grey-level differences exist between the open water and ice cover. These strong gradients occasionally pass the criteria set for identifying true ice ridges and are thus extracted during the processing.

\section{GONGLUSIONS}

The automated method of ice-ridge identification and extraction from the background ice field relied upon optimizing four parameters. (1) The detail of the ridge structure demanded by the user governs the choice of resolution of the digitized photographs. A pixel resolution of $1 \times 1 \mathrm{~m}$ discriminated the fine scale structure of individual ridge forms, but the ridge pattern was very segmented compared to the smoothed ridge pattern obtained by manual interpretation. Resampling the images at a lower resolution appears to show some success toward alleviating this problem. (2) Preprocessing the digital imagery with an edge-preserving adaptive filter eliminated much of the high frequency noise, reducing the number of errors in ice-ridge identification. (3) Setting a minimum Sobel cut-off value for the edge detection, and (4) a minimum length criteria also reduced the number of short, low-contrast linear features masquerading as ridges. Our work in the Beaufort Sea has shown that from a small representative set of digital images, optimum values for these parameters were obtained and can be applied to the entire data set.

The automated ridge-extraction procedure applied to digital images provides the analyst with a means of acquiring quantitative ridge characteristics. Preliminary results described in this study indicate a possible positive correlation between actual spot heights and their equivalent pixel edge strength. Future work requires investigating different heuristic methods of line linking for a better approximation of ridge length and reduction in the number of false-ridge identifications. Once these methodological questions are dealt with, this automated procedure offers a very rapid and efficient means of extracting two-dimensional information on ice ridging not only from aerial photography but also from SAR and other digital images.

The ridge-extraction algorithm used in this study provides a reliable estimate of the statistical distribution of ridge azimuth directions. In the Beaufort Sea transect examined, ice-ridge development is not isotropic but has a dominant east-southeast preferred orientation that is generally parallel to the local coastline. These observations suggest that large-scale or long-lived mechanisms control the ridging process. The two-dimensional analysis may provide some important insights towards understanding the dynamics of ice ridging and, at the very least, is useful for determining the appropriate direction for one-dimensional studies to be made: orthogonal to the principal ridge direction, in this case about $\mathrm{N} 25^{\circ} \mathrm{E}$. The circular histogram of ridge directions can be generated from digital images, providing an estimate of the ridge anisotropy. As an immediate and practical tool, rapidly derived directional data supplies commercial navigation with information necessary for selecting a least-energy path through the ice fields.

\section{ACKNOWLEDGEMENTS}

This investigation was supported through an NRC contract awarded to J. Lewis. Lawrence Houston provided valuable computer assistance and implemented the digital scanning procedure. Xiao Ren-ming is thanked for skillfully producing the manual ridge interpretation. P. Budkewitsch gratefully acknowledges the support of a Max Bell Fellowship in Northern Studies during the course of this work. Critical reviews by an anonymous referee helped improve the manuscript. 


\section{REFERENCES}

Ballard, D. H. and C. M. Brown. 1982. Computer vision. Englewood Cliffs, NJ, Prentice-Hall.

Davis, L. S. 1975. A survey of edge detection techniques. Comput. Graph. Image Proc., 4, 248-270.

Eppler, D. T. and L. D. Farmer. 1991. Texture analysis of radiometric signatures of new sea ice forming in Arctic leads. IEEE Trans. Geosci. Remote Sensing, 29(2), 233-241.

Giardina, C. R. and E. R. Dougherty. 1988. Morphological methods in image and signal processing. Englewood Cliffs, NJ, Prentice-Hall.

Kasvand, T. 1974. Iterative edge detection. Comput. Graph. Image Proc., 4, $279-286$.

Lewis, J. E. and P. Budkewitsch. 1993. Automated extraction of ice ridge structures in the Beaufort Sea. Ottawa, National Research Council, internal report.

Martelli, A. 1972. Edge detection using heuristic search methods. Comput. Graph. Image Proc., 1, 169-182.

Newton, K. G. and P. Budkewitsch. 1989. Lithotectonic domain identification by statistical analysis of visually and computer-aided edge-detected lineaments: a case study from Cape Smith tectonic belt, New Quebec. In Proceedings of the Seventh Thematic Conference on Remote Sensing for Exploration Geology, Calgary, Alberta, Canada, October 2-6. Ann Arbor, MI, Environmental Research Institute of Michigan, $861-875$.

Newton, G. and B. Kendrick. 1990. Image processing in taxonomy. Sydowia, 42, 246-272.

Robin, P.-Y. and E. C. Jowett. 1986. Computerized density contouring and statistical evaluation of orientation data using counting circles and continuous weighting functions. Tectonophysics, 121, 207-223.

Samadani, R. and J. F. Vesecky. 1990. Finding curvilinear features in speckled images. IEEE Trans. Geosci. Remote Sensing, 28, 669-673.

Similä, M., M. Leppäranta, H. B. Granberg and J. E. Lewis. 1992. The relation between SAR imagery and regional sea ice ridging characteristics from BEPERS-88. Int. f. Remote Sensing, 13(13), 2415-2432.

Vesecky, J.F., M. P. Smith and R. Samadani. 1990. Extraction of lead and ridge characteristics from SAR images of sea ice. IEEE Trans. Geosci. Remote Sensing, 28(4), 740-744.

The accuracy of references in the text and in this list is the responsibility of the authors, to whom queries should be addressed. 